\title{
Gender-Based Expectations and their Effect on Mental Health Amongst Black African Immigrant Young Men Living in Canada
}

\author{
Omolola Olawo
}

Cite as CJCND 2019, 1(1), pp. 82-90

\begin{abstract}
Black African immigrant men's mental health is relatively understudied. This article is part of a larger study that explored the perceptions of mental health amongst African immigrant youth living in Canada. Using an interpretive description methodology, underpinned by an intersectional and critical lens, this article addresses racial, societal, and cultural expectations that could have an effect on Black African immigrant men's mental health. Eight men and women who self-identified as Black African immigrants between the ages of 18 and 25 participated in the overall study, while five participants contributed the data for this article. Masculinity as a determinant of health, the resilience of African men, and the intersections of identity and vulnerability are discussed. It is concluded that the stigma surrounding Black African men speaking out about their mental health warrants a deeper examination in relation to their mental health outcomes. Areas of further inquiry include exploring mental health service utilization amongst Black African immigrant men.
\end{abstract}

Keywords: African men's mental health; Black men's mental health; Intersectionality; Hypermasculinity; Racial experiences

Mental health and illness are conceptualized and experienced differently across and within continents based on unique cultural, religious, social, and political factors. In some African countries, mental health care and literacy are not often considered as important due to multiple competing priorities the continent faces. This includes poverty, economic crises, communicable diseases, and internal conflicts (Gureje \& Alem, 2000; Okasha, 2002, Monteiro, 2015). Unfortunately, some distressed political climates can cause internal displacement, hunger crises, and diseases that could lead to post-traumatic stress disorder, depression, and other mental illnesses
(Amuyunzu-Nyamongo, 2013). People can find themselves in circumstances that are not conducive to their mental health, and this is complicated by the lack of mental health support from family, their communities, and the health care system in general. This coupled with the influence of traditional beliefs in supernatural causes of illnesses can impact the perception and attitudes about mental health and the acknowledgement of mental illness (Gureje \& Alem, 2000).

In African communities and in the diaspora, mental health and mental illness are highly stigmatized and those living with a mental illness, particularly those

Corresponding Author: Omolola Olawo, RN, MScN, SE Health, 25 Centurian Drive, Markham ON L3R 5N8

Email: Lola.olawo@gmail.com 
with psychotic disorders, are often feared and avoided and, in some cases, receive anger from those who encounter them (Amuyunzu-Nyamongo, 2013). A study conducted in Uganda showed that the term "depression" was not socially accepted within the population (Amuyunzu-Nyamongo, 2013). Respondents of a survey conducted in Nigeria mostly attributed the cause of mental illness to drug misuse, divine wrath/will of God and witchcraft/spiritual possession while few respondents cited family and socio-economic factors as contributing factors (Amuyunzu-Nyamongo, 2013). Other studies have also identified personal failure or punishment, stress, and trauma as perceived causes of mental illness in African societies (Gureje, Lasebikan, EphraimOluwanuga, Olley \& Kola, 2005; Monteiro, 2015). The social implications of mental illness have a particularly negative impact in African societies and this has caused what Amuyunzu-Nyamongo (2013) describes as "a silent epidemic." Families often hide members who have either been diagnosed with mental illness or are experiencing mental health challenges for fear of social discrimination (AmuyunzuNyamongo, 2013). Thus, the issues surrounding mental health/illness in African communities include acknowledgment of the illness, mental health literacy, stigma, cultural beliefs, and access to care.

In the African diaspora, many of these beliefs and the social stigma associated with mental illness or mental health challenges are present. This can lead to disparaging comments and actions from the community towards those who experience mental health challenges (Amuyunzu-Nyongo, 2013; Barke, Nyarko \& Klecha, 2010; Guruje et al., 2005). Although there is not much research on African immigrant groups living in Canada, studies conducted in the United States and Canada have found similar interconnections between religious/spiritual beliefs and mental health amongst African immigrant groups (Adekeye, Kimbrough, Obafemi \& Strack, 2014; Fenta et al., 2006). While there is a gap in research on African immigrants in Canada, there is an even larger gap where youth are concerned. It is not known how African immigrant youth perceive mental health or mental illness or their experiences with mental health challenges. This formed the basis of inquiry out of which this article emerged. The study sought to explore the attitudes, beliefs, and practices concerning mental health amongst African immigrant youth living in Canada. A recurring theme in the original study related to the beliefs about mental health amongst African immigrant young men.

Although the category "African" is ascribed to people of the continent, there are shared beliefs across the continent that to an extent ties them together, such as the traditional beliefs behind the cause of mental illness. The use of the term "African" to describe participants in this study is not meant to view Africa as a monolith. Rather, it points to an identity that although ascribed, African immigrants have come to own in the diaspora. It describes a community with shared migratory and social experiences that immigrants of African descent have found in order to feel a sense of belonging. In these spaces, they find "identity safety" in an environment where they feel valued, accepted, and welcomed (Madibbo, 2016). Thus, hereafter, I will be referring to this population as African immigrants as well as the subgroup of African immigrant men.

There is little research specifically focused on the mental health of Black African immigrant men in Canada and its contributing factors. Most research is done in the United States, and it broadly focuses on Black men, without the added layer of ethnicity and immigration status (Sellers, Bonham, Neighbors \& Amell, 2009; Watkins, Walker \& Griffith, 2010; Watkins \& Neighbors, 2007). The research that has been conducted provides us with some insight into the mental health experiences of Black men and how they perceive it. Learnings from the current literature include:

1. Racial discrimination has an effect on the mental health of Black men;

2. There are alternative expressions of mental health used by Black men that may be overlooked by health care practitioners; and

3. The idea that Black men do not have mental health problems because they are "stronger" than individuals from other races (Sellers et al., 2009; Watkins \& Neighbors, 2007; Watkins et al., 2010).

The third point was a theme present in both men and women in this study, and this may be attributed to race and ethnicity, rather than strictly gender. Research in Canada has looked at men's mental health and racial disparities in health outcomes, but not specifically African immigrant men's mental health (Evans, Frank, Oliffe \& Gregory, 2011; Toronto Public Health, 2013). 


\section{PURPOSE}

The mental health of African immigrant youth living in Canada is not well understood. Given the limited research on African immigrant youth's mental health in Canada, the original study sought to examine the attitudes towards and beliefs concerning mental health amongst this population, as well as the factors that influence their mental health outcomes. Within this youth group, gender differences also play an important role in mental health beliefs. This paper looks at the findings related to the mental health beliefs of African immigrant male youth living in Canada, specifically, Black African immigrant male young men. It addresses the questions: How do African immigrant young men living in Canada express their mental health and what are the factors that influence both their mental health as well as their ability to express their mental health? Although it was a relatively small sample, their voices lend to the limited body of work in this field.

\section{METHODOLOGY}

Thorne's (2016) Interpretive Description was used as the methodology for this study. Interpretive description ventures into the "so what" domain that seeks to develop knowledge that informs practice. In addition, its foundational assumptions were consistent with those underpinning this study, namely, a) that reality is not fixed and can involve multiple constructed realities that may contradict each other; and b) that value is placed on subjective and experiential knowledge as a source of insight regarding practice (Thorne, 2016).

The chosen theoretical perspectives were critical theory and intersectionality. Critical theory was chosen for its emphasis on critique of dominant power structures and social inequality that may negatively impact one's health. Research from a critical perspective is not only concerned with disrupting the status quo and challenging oppressive structures but also with empowering others and helping those with limited power either acquire or reclaim it (Willis, 2007). Intersectionality is influenced by critical theory, and it advances that people's experiences are the results of the intersections or interconnections of two or more of their identities (Hankivsky \& Christoffersen, 2008). It also acknowledges that people's identities are interconnected socially, politically, and ideologically, and they work together to bring about positive or negative outcomes (Gustafson, 2005). These two theoretical perspectives were foundational to the study, as they focused on uncovering potential causes of health inequities that African immigrant youth might experience.

\section{Participant Recruitment}

Study participants between the ages of 18-25 from Toronto, Canada were recruited through purposive and snowball sampling. The participants were sought through various avenues such as a university African Students Association, a local church, and word of mouth. A sample size of 7-10 was planned and eventually eight participants were interviewed. This range was chosen because of the intent to conduct an in-depth exploration of the topic from the point of view of those who are a part of the community of African immigrant youth. Feasibility was also a consideration because of time constraints related to this project (master's thesis). It was determined that the sample size of eight participants was sufficient after conducting interviews and no new information was emerging from participants. Regarding male mental health experiences, five participants provided input. Two of these participants were male and offered direct experiences, while the other three female participants offered input based on men in their lives or what they had noticed growing up. Participants were included if they were born in an African country, had come to Canada during grade school years (between the ages of 5 and 18), and were currently living in Canada. Current hospitalization related to a mental health crisis was an exclusion criterion for this study.

\section{Data Collection}

Data collection took place between November 2017 and February 2018. Both telephone $(\mathrm{N}=4)$ and inperson $(\mathrm{N}=4)$ semi-structured interviews were conducted. Open-ended questions were asked, including questions related to participants' beliefs about mental health/illness. Some questions included: "How has your culture influenced your views on mental health?" "What situations or experiences have you gone through that have influenced your views on mental health?" "Do you believe there is a stigma associated with the term 'mental health' amongst Africans" "Do you think there is a difference between the way mental health is perceived in African men and African women?" Interviews were audio recorded and transcribed immediately afterward, as data analysis 
was done concurrently with the interviews. This allowed revision of interview questions based on data that had emerged in the early parts of the study. Member checking was done with study participants in order to share with them the synthesis of what was gained from their contributions and to allow them to reflect on the extent to which it represented their experiences. These member checks served as an opportunity to check the truth value of the interpretation of the data. Informed consent was obtained from each participant, and process consent was utilized throughout the interviews. Participants were offered a $\$ 10$ cash gift as a token of appreciation for taking part in the study. All participants were provided pseudonyms to protect their identity and these were used in interview transcripts, the data analysis process, as well as this article. Ethics approval for the study was obtained through York University's Office of Research Ethics.

\section{Data Analysis}

Thorne's (2016) suggestion of organizing data into indeterminate or undefined categories was used to avoid constraining the data into a rigid structure. Four different categories, labelled A, B, C, and D were used to place similar ideas from the transcripts. During thematic analysis, information in the data with similar content or meanings were explored, as well as information that differed from one participant to another. This was an attempt to determine what themes were common amongst the group and what ideas were unique to particular participants. A concept map provided a visual representation of relationships that were starting to take shape, and linkages were made between and within categories. Several themes emerged by way of this process, one being mental health experiences based on gender, particularly men. This served as the basis for this paper.

\section{Demographic Profile}

The overall study sample included eight participants between the ages of 18 and 25, six of whom were female and two were male. Source countries included Nigeria ( $\mathrm{N}=4)$, Ghana ( $\mathrm{N}=1)$, Ethiopia $(\mathrm{N}=1)$, Zambia $(\mathrm{N}=1)$ and Gambia $(\mathrm{N}=1)$. All participants identified as Black Africans from various ethnic groups. They arrived in Canada at different ages (between 5 and 18). Five participants immigrated to Canada with their family, while three came by themselves, as international students. Three participants had completed a bachelor's degree and the other five had completed some college or university.

\section{FINDINGS}

\section{Gender-Based Beliefs and Expectations Related to Mental Health}

Although there were only two male participants in the study, some of the women spoke about male figures in their lives and their experiences. Regarding genderbased beliefs, both male participants readily related their mental health to the completion or achievement of goals that they had set out for themselves. One participant (David) stated:

My mental health revolves around me
meeting my goals. I don't really ever focus
on how I'm feeling. My feelings are
dependent on goals. So, if I'm meeting those
goals, I'm feeling great. If I'm not meeting
those goals, I'm not feeling great. (David,
age 25)

The second male participant also echoed similar thoughts. He said:

\section{For me, I'd say that mental health is related to some sort of goal or plan. Or sort of an idea of what I should be doing or looking forward to. That's how I see it. (Dami, age 20)}

They both stated that in their experiences, men were expected to be emotionally "stable" when dealing with challenges in life. Men were expected to keep a "level head," especially when others were watching, and only in private, they could "break down."

According to five of the participants, men were not expected to speak up about their feelings. One participant, Felicia, spoke about her expectations of men based on her cultural norms. She said:

$$
\begin{aligned}
& \text { "For my Nigerian side, I've just always } \\
& \text { expected for guys especially to always be } \\
& \text { strong. To never cry, or never really open up } \\
& \text { too much. So that's what I've always } \\
& \text { expected from guys. Just in terms of how } \\
& \text { Nigerian men are." (Felicia, age 24) }
\end{aligned}
$$

Regarding speaking about mental health and whether or not a difference exists between men and women, Dami stated: 
I'd say guys don't really talk about it if anything. I'll say I've talked about it more with girls than I've talked about it with guys. Maybe I don't talk about it with guys or guys just don't talk about it with other guys, or they don't talk about it with me. I'd say that girls just generally talk about that more in my experience... But I think that guys don't share much to begin with. (Dami, age 20)

Another female participant, Sarah, spoke about a Nigerian male friend of hers who shared with her his thoughts about speaking to others. She stated:

\begin{abstract}
We were just talking about when people confide in each other. And he was saying that he would never do that, and I was like, why can't you just open up and talk to people? And he's like, "why would I talk to people about my problems? I'm just putting it on them. Like that's private. Why would I ever say that?" And then I was like, that's kind of the backbone of communication. And we were going back and forth. And another guy came and agreed with him. And I also think that you have to also separate genders. Because I think girls find it easier to talk about it. And a guy was saying this. Like I was against talking about it before too, but how against it he was surprised me. (Sarah, age 19)
\end{abstract}

She also mentioned that her brother had gone through difficult times and only recently acknowledged this to her. She stated:

You know, I didn't realize, like I had just spoken to my brother some months ago, and I literally did not know he was going through anything. Until he had finally opened up to me years and years later. Only when I kind of forced him to. And it was in conversation that he had brought it up. Like he just kept it in all of these years and did not want to talk about it.

Both men in this study acknowledged that conversations amongst their friends could be largely superficial and general, and conversations about emotions or feelings rarely took place. Several participants stated that they felt it was much easier for women to speak up about their feelings.

\section{Racial and Societal Expectations for African Immigrant Men}

The participants' gender related beliefs are intertwined with racial and societal expectations. David spoke in depth about his experience as a Black male, and how that identity influenced his beliefs. He stated that the experiences in society that Black people have gone through-and are currently going through-especially Black men, build them to be strong and leaves little room for "weakness." Regarding work, he said:

I know sometimes mental health says it's ok to be vulnerable or breakdown at certain points you don't feel good. But the society I'm in, especially the industry I'm in, the whole corporate environment. As a Black man and the challenges I have to go through, you really have to build a wall to protect yourself to advance you to the next level because there are things that are said in the workplace that may necessarily offend you...I mean I can stand up for that, in the corporate culture, but like it's only as far as it can take me. So, imagine me standing up for that every time it happens. I almost become like a nuisance, right? (David, age 25)

Regarding how race affects one's mental health, he stated:

How do I think it can affect mental health? It basically asks the question, "Why should you be weak?" There's no reason for you to be weak. If certain people have gone through the struggle or way more struggle than you've gone through, and you as a Black person want to be weak?... And if you know, ancestors or whatever went through harder struggles especially in North America, you have people that went through slavery, and then you want to complain that your struggles of not being able to make your rent money are a lot? I mean, it asks that question. (David, age 25)

Another participant, Thandi, mentioned that she has had to constantly encourage her younger brother to speak up about his experiences, especially because he is a Black male living in a predominantly White neighborhood and in a Zambian household. She said: 
I'll be like, "you better talk to me, what's going on? You can always talk to me". So, it's like at least having that environment with me where he feels like ok, whatever is going on, because he's gonna have his own experiences too, and he might not know how to articulate it ... He's a Black male in a predominantly White neighborhood where we live at. He doesn't have like a close Black friend, so there's certain experiences that he's not able to share with those friends, right? So, at least being able to talk to him and tell him "this happens, that happens, this is life. If anything, whenever, talk to me." I think we need to foster that kind of environment. I feel like certain African parents are just like, you're the general of the house and you just give orders and that's it. That's not really conducive to like a good place for someone to maintain good mental health. So just that openness. We need to let go of some of these traditions and cultural nonsense that make us interact the way that we do. (Thandi, age 25)

\section{DISCUSSION}

\section{The Impact of Racial and Ethnic Identities on Mental Health}

Before discussing intersectional identities and their influence on mental health, it is important to distinguish the difference between race and ethnicity. Although they are often used interchangeably, and they are both socially constructed, they are not the same. Markus (2008) offers a definition of race and ethnicity, where race is defined as a set of ideas and practices that "associates differential value, power, and privilege with [physical and behavioural] characteristics and establishes a social status ranking among different groups" (p. 654). Ethnicity, on the other hand, "allows people to identify or to be identified with groupings of people on the basis of presumed (and usually claimed) commonalities including language, history, nation or region of origin, customs, ways of being, religion, names, physical appearance, and/or genealogy or ancestry" (p. 564). Using the participants in the study as examples, they all identified their racial category as Black. But their ethnicities differ based on the region of the continent wherefrom they came. It is also important to note that national identity also differs from race and ethnicity.
Within a nation, multiple racial and ethnic categories could exist.

African immigrant men might be particularly susceptible to mental health challenges because of the intersectionality of their race, ethnicity, and gender. While discussing race, David touched on the societal expectations put on a Black man and the relationship between Black men and women. He stated that Black men go through unique experiences that build them to be strong saying, "with the way life is made, especially as a Black person, you have to be strong." He used the examples of working "twice as hard" as others in a classroom or workplace setting in order to be recognized or having to be the best at a sport because of his Blackness. He stated that although these experiences can build people to be strong, they can also affect their mental health, because it begs the question, "why be weak?" This further ties into the expectation of being able to overcome anything that comes a person's way. He went on to state that he had noticed Black men being called "weak" by Black women in some relationships and because of this, some men do all they can to maintain the role of "the strong Black man." This also intertwined with his cultural expectations to "keep a level head and weather the storm." He stated: "Where I'm coming from [in Nigeria], I feel you're really molded to not let society impact you in a negative way."

Felicia, another participant, touched on cultural expectations for African men. She mentioned that because of her experiences and her cultural norms, she expected men to "always be strong; to never cry, or never really open up too much." All the participants in the study noted that from what they have noticed, people of African backgrounds do not speak up about their mental health because of the stigma associated with it. Sarah, Felicia, and Dami also mentioned that men in particular do not really speak up much about their mental health. Felicia went as far as saying if there was an event promoting mental health in the African diaspora, "there will be no boys there for sure." This could be because opening up about one's mental health requires a level of vulnerability. For one to be vulnerable means that they are "capable of being physically or emotionally wounded" (MerriamWebster, 2018, p. ?). For African men, this could translate as being put in a position of weakness that they have been brought up to avoid.

In order to protect against being perceived as weak they may exhibit hypermasculinity, an exaggerated 
form of masculinity, in which they display a stereotypical "gendered display of power and consequent suppression of signs of vulnerability" (Spencer, Fegley, Harpalani \& Seaton, 2004, p. 234). Some women's expectations of men to be strong and stoic can cause men to fear being emasculated if they open up about their emotional health. Hypermasculinity can then become a coping mechanism for them if they feel they are at risk of being emasculated by women or even other men. Although it may protect them from the immediate fear or threat of weakness, in the long run, the internalization of hypermasculinity places them in a particularly vulnerable state because they may be suffering in silence (Barker, Ricardo, Nascimento, Olukoya \& Santos, 2010; Spencer et al., 2004; Watkins \& Neighbors, 2007).

David offered another example of his experience at work which speaks to a power difference that exists in workplaces and could further affect one's mental health. His choice not to speak out against inappropriate situations so as to not be labelled a "nuisance" is unfortunately a process that perpetuates racial inequalities in workplaces. Having to silence himself while dealing with racialized interactions in order to advance in his career only reinforces the power imbalance that already exists in society. All of this in combination with immigrating at a young age to a country where one is a minority can have a negative effect on one's mental health.

\section{Masculinity as a Determinant of Mental Health}

For men, masculinity could serve as a determinant of mental health and in most societies, hegemonic masculinity could be idealized in the hierarchy of masculinities (Evans et al., 2011). Evans et al. explain what hegemonic masculinity looks like in most Western societies: "White, heterosexual and middle class, and possessing stereotypical masculine traits of assertiveness, dominance, control, physical strength, and emotional restraint" (p. 8). Those who do not meet the ideal standard of masculinity could be marginalized as a result. It is no wonder that men in this study held the control over the achievement of their goals dearly to them, as well as the ability to maintain control over their emotions. When they were confronted with situations and challenges that were not in their control, they described their mental health as poor. For example, Dami who was thrown for a loop when he had an internship cancelled after he had made plans for his semester and David who stated he had to constantly control his emotions no matter the situation, especially to the outside world. This would also explain the expectations and norm for men to not show outwardly expressions of emotions, particularly if they have been conditioned to maintain stereotypical masculine traits.

While the expectations of hegemonic masculinity might cut across all societies, the consequences for not fitting into that category may differ between cultures and racial categories. For African men, when their maleness is combined with their race, ethnicity, immigrant status and cultural expectations, we can see men who are at a higher risk for poor mental health outcomes. Like the participants in the study mentioned, the cultural expectation to always maintain strength in the face of adversity and to let go of all signs of weakness, such as crying, can lead them to be even more vulnerable to mental health challenges. It could create an emotional crisis as their identities as both African and Black men could be challenged. As David said, "why be weak?"

\section{Resiliency in African Immigrant Men}

The notion of resilience has applicability within the context of African peoples mental well-being. The idea of inner strength was reflected through several participants' accounts. There was the belief that they were "built to be tough," to "always overcome," and to always have hope no matter their situations. This internal strength seems to hold one up, especially when comparing their situation to others who may be worse off than they are. The relation of past situations to present struggles and the experience of adversity to build resilience in African youth is consistent with other research (Lothe \& Heggen, 2003; Theron \& Theron, 2013). It is important to note that although comparing struggles can help bolster resilience, it can also serve as a double-edged sword that becomes a barrier to seeking help for mental health issues. If there is an expectation for a person to always have hope and be strong in the face of adversity, there may be a fear of being looked down upon if they were to show signs of helplessness or defeat. This is especially true for men, when considering the intersections of their gender, cultural expectations and identity, immigrant identity, as well as societal expectations.

Despite the influence of the participants' cultures on their beliefs about mental health, they were still able to acknowledge that it is important to speak out about challenges that they were experiencing. Even if they 
could not talk to other men about their situations, both men in the study acknowledged that they speak to one or two women in their lives about challenges they were experiencing. They also encouraged that other men find people they could confide in and speak up about their mental health. They were able to recognize the need to share that burden with someone else when necessary. There was also a recognition of a need for a shift in the cultural mindset around mental health. This in itself is a resilient trait. Thandi mentioned that she fostered an open environment for her brother to be able to share his thoughts and feelings with her. It is important that these safe spaces exist for African young men to gain support from one another.

\section{CONCLUSION}

Though this paper contributes to filling in a large gap in the research of Black African men's mental health, there is still a long way to go. Future research could dive into multigenerational experiences of mental health: Adolescents, young adults, and older adults; mental health service utilization amongst Black African immigrant men; and help-seeking methods for Black African men experiencing mental health crises.

The racial, societal, and cultural expectations placed on Black men can place them at a risk for poor mental health outcomes. The stigma around speaking about their mental health and help seeking in their communities, along with the expectations of the women in their lives perhaps warrant deeper examination of their mental health outcomes. It is of importance to create open and safe environments where conversations around mental health can take place without African immigrant men rendered vulnerable. Programs and services that cater to men's mental health also need to consider how intersections of men's identities place them at higher risks of mental health challenges and these should be reflected in how they deliver care to African immigrant men.

\section{REFERENCES}

Adekeye, O., Kimbrough, J., Obafemi, B., \& Strack, R. W. (2014). Health literacy from the perspective of African immigrant youth and elderly: A photo voice project. Journal of Health Care for the Poor \& Underserved, 25(4), 1730-1747. doi:10.1353/hpu.2014.0183

Amuyunzu-Nyamongo, M. (2013). The social and cultural aspects of mental health in African societies. Commonwealth Health Partnerships,
59-63. Retrieved from

http://www.commonwealthhealth.org/wpcontent/uploads/2013/07/The-social-andcultural-aspects-of-mental-health-in-Africansocieties_CHP13.pdf

Barke, A., Nyarko, S., \& Klecha, D. (2011). The stigma of mental illness in Southern Ghana:Attitudes of the urban population and patients' views. Social Psychiatry and Psychiatric Epidemiology, 46(11), 1191-1202.

Barker, G., Ricardo, C., Nascimento, M., Olukoya, A., \& Santos, C. (2010). Questioning gender norms with men to improve health outcomes: Evidence of impact. Global Public Health, 5(5), 539-553.

Evans, J., Frank, B., Oliffe, J. L., \& Gregory, D. (2011). Health, illness, men and masculinities (HIMM): a theoretical framework for understanding men and their health. Journal of Men's Health, 8(1), 7-15.

Fenta, H., Hyman, I., \& Noh, S. (2006). Mental health service utilization by Ethiopian immigrants and refugees in Toronto. Journal of Nervous \& Mental Disease, 194(12), 925-934. doi: 10.1097/01.nmd.0000249109.71776.58

Gureje, O. \& Alem, A. (2000). Mental health policy development in Africa. Bulletin of the World Health Organization, 78(4), 475-482. Retrieved from http://www.who.int/bulletin/archives/78(4)475. pdf

Gureje, O., Lasebikan, V. O., Ephraim-Oluwanuga, O., Olley, B. O., \& Kola, L. (2005).

Community study of knowledge of and attitude to mental illness in Nigeria. British Journal of Psychiatry, 186(5), 436-441.

Gustafson, D. (2005). Transcultural nursing theory from a critical cultural perspective. Advances in Nursing Science, 28(1), 2-16.

Hankivsky, O., \& Christoffersen, A. (2008). Intersectionality and the determinants of health: A Canadian perspective. Critical Public Health, 18(3), 271-283. Doi: $10.1080 / 09581590802294296$

Lothe, E. A., \& Heggen, K. (2003). A study of resilience in young Ethiopian famine survivors. 
Journal of Transcultural Nursing, 14(4), 313320 .

Okasha, A. (2002). Mental health in Africa: the role of the WPA. World Psychiatry, 1(1), 32.

Markus, H. R. (2008). Pride, prejudice, and ambivalence: Toward a unified theory of race and ethnicity. American Psychologist, 63(8), 651.

Monteiro, N. M. (2015). Addressing mental illness in Africa: Global health challenges and local opportunities. Community Psychology in Global Perspective, 1(2), 78-95.

Sellers, S. L., Bonham, V., Neighbors, H. W., \& Amell, J. W. (2009). Effects of racial discrimination and health behaviors on mental and physical health of middle-class African American men. Health Education \& Behavior, 36(1), 31-44.

Spencer, M. B., Fegley, S., Harpalani, V., \& Seaton, G. (2004). Understanding hypermasculinity in context: A theory-driven analysis of urban adolescent males' coping responses. Research in Human Development, 1(4), 229-257.

Theron, L. C., \& Theron, A. (2013). Positive adjustment to poverty: How family communities encourage resilience in traditional African contexts. Culture \& Psychology, 19(3), 391- 413.

Thorne, S. (2016). Interpretive description: Qualitative research for applied practice. 2nd ed. California: Left Coast Press Inc. Routledge.

Toronto Public Health. (2013). Racialization and Health Inequities in Toronto. Retrieved from https://www.toronto.ca/legdocs/mmis/2013/hl/b grd/backgroundfile-62904.pdf

Watkins, D. C., \& Neighbors, H. W. (2007). An initial exploration of what 'mental health' means to young black men. The Journal of Men's Health \& Gender, 4(3), 271-282.

Watkins, D. C., Walker, R. L., \& Griffith, D. M. (2010). A meta-study of Black male mental health and well-being. Journal of Black Psychology, 36(3), 303-330.

Willis, J. W. (2007). Foundations of qualitative research: Interpretive and critical approaches. Thousand Oaks, CA: SAGE Publications Ltd doi: $10.4135 / 9781452230108$ 